\title{
The Effects of Aceclofenac and Nabumetone in Osteoarthritis
}

\author{
Paul S, ${ }^{1}$ Das N, ${ }^{2}$ Ghosh $\mathrm{S}^{3}$ \\ 'Department of Pharmacology, Medical College, Kolkata, West Bengal, India, ${ }^{2}$ Department of Pharmacology, NRS Medical Col- \\ lege, Kolkata, West Bengal, India, ${ }^{3}$ Department of Orthopedics, CN Medical College, Kolkata West Bengal, India
}

\section{ABSTRACT}

Introduction: Aceclofenac, a NSAID is widely used in the treatment of pain and inflammation associated with osteoarthritis. Nabumetone, a recently developed preferential cyclo-oxygenase 2 inhibitor has also proved to be equally effective. The present study was undertaken to evaluate the 'real better' drug, amongst these with better efficacy and gastro-intestinal tolerability as well.

Methods: Four hundred and twenty-three patients of either sex, aged 40-64 years with uncomplicated osteoarthritis of knee joint were randomly allocated into three equal groups receiving aceclofenac, nabumetone or placebo. A baseline pain measurement was done with Visual Analogue Scale (VAS: 0-10 scale) and Investigator Global Assessment of Disease status (IGADS: 0-4 point scale). Code was broken at the end of two weeks or earlier to eliminate any real fatal outcome. Final evaluation of efficacy was done at the end of four weeks. The significance of difference between the treatment outcomes was analyzed using one way ANOVA test.

Results: During the active comparator controlled period, the most common reason for discontinuation was unacceptable adverse events. While 108 (76.6\%) participants could take the full course of treatment with aceclofenac, $118(83.7 \%)$ of the nabumetone group completed the study. Drop outs were highest in the placebo group (33.9\%) followed by the aceclofenac group $(12.1 \%)$ and nabumetone group $(8.5 \%)$. Discontinuation due to G.I. intolerance was least in the placebo group $(2.1 \%)$ followed by the nabumetone group (5\%) and aceclofenac group (7.8\%).

Conclusions: The preferential inhibition of cyclo-oxygenase 2 by nabumetone was postulated to afford better clinical efficacy and gastrointestinal tolerability in osteoarthritis as compared to aceclofenac. 


\section{INTRODUCTION}

Pain associated with osteoarthritis (OA), a slowly evolving articular degenerative disease is commonly treated with nonsteroidal anti-inflammatory drugs (NSAIDs), both nonselective and cyclo-oxygenase 2 (COX-2) selective inhibitors. ${ }^{1}$ Aceclofenac, a non-steroidal antiinflammatory drug is widely used in the treatment of pain and inflammation associated with osteoarthritis. Nabumetone, a recently developed preferential cyclooxygenase II inhibitor has also proved to be effective in treatment of osteoarthritis. Prolonged use of NSAIDs has been limited as approximately $25 \%$ of NSAID users develop gastro-intestinal (G.I.) symptoms. ${ }^{2}$ Though the role of non-selective COX inhibitors has been generally appraised; the preferential COX-2 inhibitors are still under evaluation.

The present study was undertaken to evaluate the 'real better' drug amongst aceclofenac, a non-selective COX inhibitor and nabumetone, a recently developed preferential COX-2 inhibitor, if any, with better clinical efficacy and gastrointestinal (GI) tolerability as well in the treatment of osteoarthritis.

\section{METHODS}

A double blind placebo controlled study was conducted by the departments of Orthopaedics and Pharmacology of North Bengal Medical College in West Bengal, India, during the period June 2006-January 2007. The patients enrolled for the study were selected from the Orthopaedics Out-patients Department. The study protocol and procedures were approved by the Institutional Review Board and clearance from the Ethical Committee of the institution was obtained. Written informed consent was taken from each participant in their own language before they entered the study. Patients of either sex, (40-64years) suffering from uncomplicated Osteoarthritis (OA) of knee joint with symptoms for at least six months having fulfilled the X-Ray criteria for Kellgren's classes II or III ${ }^{3}$ and those who met American Rheumatologic Association (ARA) functional Class I, II or $\mathrm{III}^{4}$ were randomly allocated for the study. However patients with significant renal impairment (creatinine clearance $<30 \mathrm{ml} / \mathrm{min}$ ), Class III / IV angina, uncontrolled congestive heart failure (CHF), uncontrolled hypertension (BP $>160 / 100)$, clinically significant physical and mental abnormalities or abnormal laboratory examinations, hepatic disease, bronchial asthma or with history of any active G.I. bleeding, neoplasia, acute meniscus injury, or arthroscopy in the study joint within last six months, obesity (BMI $\geq 40$ ) or those allergic to conventional NSAIDs were excluded from the present study. Patients were also excluded if they required systemic steroids, warfarin, lithium, low dose aspirin, anti-ulcer drugs or intra-articular steroids, within last two months of entering the study.
The participants selected for the study entered a pretreatment period of ten days during which baseline investigations like complete haemogram, skiagram of the affected knee joint (AP, lateral and skyline view), Liver function tests (LFT), Electrocardiogram (ECG) estimation was done. Other analgesics were discontinued except paracetamol (upto $2 \mathrm{~g}$ /day for a maximum of three days but not within last 48 hours of assessment of arthritis). Baseline pain measurement was done with visual analogue scale (VAS 1-100 score), suitably modified for the present study. The investigators, blinded for the present study, were asked to assess the condition of the individual participants before and after the study in an established Investigator Global Assessment Scale (IGADS: 0-4 point scale where $0=$ very well, $1=$ moderately well, $2=$ fair, $3=$ poor, $4=$ very poor). ${ }^{5}$

Four Hundred and twenty-three patients finally entering the study were randomly allocated in a double blind technique into three equal groups of 141 each and received orally either aceclofenac (100 $\mathrm{mg})$ or nabumetone $(750 \mathrm{mg}$ ) or placebo, twice daily for four weeks. Patients were asked to come for first follow up after two weeks or earlier on appearance of any adverse symptoms like GI distress, ankle edema, any episode of upper GI bleeding etc. that warranted the end of trial. Final evaluation of efficacy was done at the end of four weeks. Code was broken once only at the end of second week of the study to eliminate any 'real' fatal outcome.

Efficacy was evaluated using the Western Ontario and Mc Master Universities OA index (WOMAC) VA 3.0 pain schedule (100-Visual Analog Scale) (VAS), suitably modified in our clinic to serve the purpose, where ' $O$ ' was marked for absence (no pain), while '10' was marked for unbearable extreme pain. $(0=$ no pain and 10 = extreme pain) and IGADS: 0-4 point scale. ${ }^{6}$

The significance of difference between the treatment outcomes was analyzed using one way ANOVA test. The $P$ value less than 0.05 was considered significant. Statistical analysis was done using Microsoft office excel 2003.

\section{RESULTS}

Four Hundred and twenty-three patients (40-64 years) suffering from uncomplicated $\mathrm{OA}$ of knee joints were enrolled into our study. Of these, 189 (44.7\%) were Male. Of 423 patients, 315 (74.8\%) completed the study. The mean age was 56.2 years in male and 51.3 years in female (Table 1). The mean duration osteoarthritis was 4.3 years.

During the active comparator controlled period, the most common reason for discontinuation was unacceptable adverse events (Table 2). While 108 
(76.6\%) participants could take the full course of treatment with aceclofenac, $118(83.7 \%)$ of the nabumetone group completed the study. Drop outs were highest in the placebo group (33.9\%) followed by the aceclofenac group (12.1\%) and nabumetone group $(8.5 \%)$. Discontinuation due to G.I. intolerance was least in the placebo group $(2.1 \%)$ followed by the nabumetone group (5\%) and aceclofenac group (7.8\%). A higher percentage of patients in aceclofenac group were withdrawn for lower extremity edema $14.2 \%$ in aceclofenac group and $3.6 \%$ in nabumetone group). The number of patients discontinuing therapy due to hypertension ( $\mathrm{BP} \geq 160 / 100 \mathrm{~mm}$ of $\mathrm{Hg}$ ) was comparable in all the groups. At the end of the second week of active comparator controlled period, tolerability of nabumetone $(83.7 \%)$ was comparable to aceclofenac $(76.7 \%)$. However the reasons for drop-outs were not followed up.

At the end of study period of 4 weeks, individual assessment of clinical improvement was done and the difference in treatment outcome (Table 3) was recorded in each group (Mean \pm S.E.), The significance of difference between the treatment outcomes was analyzed using one way ANOVA test. The $P$ value less than 0.05 was considered significant Assessment of the clinical condition as done by the investigators was analyzed (Table 4). Aceclofenac as well as nabumetone markedly improved the clinical condition in the study population. At the end of the study, only $10.2 \%$ of the aceclofenac group and $6.6 \%$ of the nabumetone group were 'very poor' responsive against the placebo group (46.8\%). Though the response in both drug groups was more or less similar throughout the study, it can be said that clinical efficacy of nabumetone was significantly higher than aceclofenac, at least in the present study by the 'blind' investigators.

\section{DISCUSSION}

Osteoarthritis (OA) is a slowly evolving articular degenerative disease characterized by gradual development of joint pain, stiffness and limitation of movement. It is the commonest form of arthritis in the middle aged and elderly population leading to pain, functional disability and significant reduction in the overall quality of life. It accounts for approximately half of all chronic conditions in persons older than 65 years of age. ${ }^{6}$

Although a variety of symptoms exist, pain is the most frequently cited reason to seek the help of a physician. Appropriate treatment of $O A$ is necessary to maintain patients' mobility and overall quality of life as it can lead to severe functional impairment. Acetaminophen and non-pharmacological approaches like exercise and measures to improve joint biomechanics can be effective first line treatment options for patients with OA. ${ }^{7}$ Since inflammation is usually not severe, smaller doses of NSAIDs may be satisfactorily used. Analgesics having significant anti-inflammatory effects may be of benefit in many patients with OA. ${ }^{8}$ NSAIDs are the most commonly prescribed agents ${ }^{9}$ for treatment of both pain and inflammation in $\mathrm{OA}$.

The NSAIDs act by inhibition of COX-II, a key enzyme involved in the pain and inflammation associated with osteoarthritis. Although nonselective NSAIDs can be effective for modifying the pain and other symptoms of osteoarthritis, the elevated risk of $\mathrm{Gl}$ toxicities associated with these agents, due to their additional inhibition of the COX isoenzyme COX-I often limits their use. ${ }^{10}$ Analgesic dose of most NSAIDs are lower than their anti inflammatory doses. However, the effects of NSAIDs on the articular cartilage and the outcome of osteoarthritis is controversial. ${ }^{11}$

COX-I and COX-II differ in their sensitivity to inhibition by certain anti-inflammatory drugs. This observation has led to the recent development of agents that preferentially inhibit COX-II which could have therapeutic advantage over conventional NSAIDs because COX-II is the predominant cyclooxygenase at sites of inflammation, whereas COX-I is the major source of cytoprotective prostaglandins in the Gl tract. The matter is not settled, but the anti inflammatory actions of these drugs are associated with improved GI tolerance compared to their nonselective counterparts in at least one trial of clinical outcome. ${ }^{12}$

Aceclofenac is approved in the United States for the long term symptomatic treatment of rheumatoid arthritis, osteoarthritis, and ankylosing spondilytis. It is the most commonly used NSAID in Europe. ${ }^{13}$ It has a more potent analgesic, antipyretic and anti-inflammatory activity than indomethacin and naproxen besides its potential in reducing intracellular concentration of frees arachidonates. ${ }^{14}$ Its potency against COXII is substantially greater than that of indomethacin, naproxen or several other NSAIDs. ${ }^{15}$ Aceclofenac may modulate $\mathrm{PGE}_{2}$ production by increasing the synthesis of interteukin-1 receptor antagonist and decreasing nitric oxide synthesis in human articular chondrocytes. ${ }^{16}$

Nabumetone is a recently developed pro-drug which generates an active metabolite 6-methoxy-2naphthylacetic acid (6-MNA) which is relative more potent COX-II inhibitor. It is an active anti-inflammatory drug that possesses antipyretic and analgesic activities. The incidence of GI ulceration appears to be lower with nabumetone than with other NSAIDs ${ }^{17}$ which may be due to the fact that nabumetone is a pro-drug and an active compound is metabolically generated only after absorption of the administered drug into systemic 
Paul et al. The Effects of Aceclofenac and Nabumetone in Osteoarthritis

Table 1. Distribution of study population according to sex and age

\begin{tabular}{llllllll}
\hline Sex & $\mathbf{4 0 - 4 4}$ & $\mathbf{4 5 - 4 9}$ & $\mathbf{5 0 - 5 4}$ & $\mathbf{5 5 - 5 9}$ & $\mathbf{6 0 - 6 4}$ & $\mathbf{N}(\%)$ & Mean age \\
\hline Male & 15 & 31 & 11 & 04 & 38 & $189(44.7 \%)$ & 56.2 \\
Female & 18 & 38 & 51 & 57 & 70 & $234(55.3 \%)$ & 51.3 \\
\hline
\end{tabular}

Table 2. Adverse events in placebo, aceclofenac (100 mg b.i.d.) and nabumetone (750 mg b.i.d) groups (Causes for discontinuation of therapy)

\begin{tabular}{llll}
\hline Group & G.I side effects & Ankle edema & Hypertension \\
\hline Placebo & $3(2.1 \%)$ & $0(0 \%)$ & $2(1.4 \%)$ \\
Aceclofenac & $11(7.8 \%)$ & $3(2.1 \%)$ & $2 .(1.4 \%)$ \\
Nabumetone & $7(5 \%)$ & $2(1.4 \%)$ & $2 .(1.4 \%)$ \\
\hline
\end{tabular}

Table 3. Effect of treatment of osteoarthritis with placebo, $100 \mathrm{mg}$ aceclofenac (twice daily) and $750 \mathrm{mg}$ nabumetone (twice daily) after 4 weeks

\begin{tabular}{llll}
\hline DRUG & $\mathbf{n}$ & Mean \pm S.E & p value \\
\hline Placebo & 89 & $0.79 \pm 0.15$ & $<.0001$ \\
Aceclofenac & 108 & $3.79 \pm 0.17$ & \\
Nabumetone & 118 & $4.23 \pm 0.13$ & \\
\hline
\end{tabular}

Table 4. Disease status of the study population (IGADS: $0-4$ point scale)

\begin{tabular}{llllll}
\hline Drug week & $\mathbf{0}$ & $\mathbf{1}$ & $\mathbf{2}$ & $\mathbf{3}$ & $\mathbf{4}$ \\
\hline Placebo & $2(2.2 \%)$ & $5(5.6 \%)$ & $7(7.7 \%)$ & $35(39.3 \%)$ & $40(44.9 \%)$ \\
Aceclofenac & $14(13 \%)$ & $27(25 \%)$ & $42(38.9 \%)$ & $13(12 \%)$ & $12(11.1 \%)$ \\
Nabumetone & $18(15.3 \%)$ & $37(31.4 \%)$ & $43(36.4 \%)$ & $11(9.3 \%)$ & $9(7.6 \%)$ \\
\hline
\end{tabular}

* $0=$ Very Well, 1 = moderately Well, 2 = Fair, 3 = Poor, $4=$ Very Poor

circulation. Selective inhibition of cycloxygenase is unlikely since the active metabolite of nabumetone (6MNA), is not a selective inhibitor of COX-II. It exhibits a reduced risk of $\mathrm{GI}$ toxicity when compared with aceclofenac $^{18}$ as well as other non selective NSAIDs. ${ }^{19}$ Its clinically important anti-inflammatory and analgesic efficacy in the treatment of acute and chronic pain and its favorable safety and tolerability profile in OA have been reviewed elsewhere. In a previously reported 2part, 14-week placebo and active comparator controlled trial, nabumetone, (1-2 g once a day) demonstrated clinical improvement that was significantly superior to placebo and comparable to aceclofenac $(100 \mathrm{mg}$ b.i.d) in patients with OA in three months of therapy. ${ }^{20}$ The present study indicates that nabumetone, in equal effective dose, produced statistically significant improvement of the clinical conditions of OA and less GI adverse events than aceclofenac,in the present study population which corroborates with the impression of other studies.

\section{CONCLUSIONS}

The present study shows that in equal effective doses, nabumetone (750mg twice dally) provides better clinical efficacy and lesser GI side effects as well in OA as compared to Aceclofenac. Long term study of efficacy and adverse events due to both the study drugs may be conducted for further valuation.

\section{REFERENCES}

1. Hochberg MC, Dougados M. Pharmacological therapy of osteoarthritis. Best Pract Res Clin Rheumatol. 2001;15:583-93.

2. Larka EN, Smith JL, Lidsky MD, Sessons SI, Graham DY. Dyspepsia in NSAID users, the size of the problem. J Clin Gastroenterol. 1998;11:158-62.

3. Kellgren JH, Lawrence JS. Radiological assessment of osteoarthritis. Ann Rheum Dis. 1957;16:494-502.
4. Gottesdiener K, Schnitzer T, Fischer C, Bockow B, Markenson $\mathrm{J}$, et al. Results of a randomized dose ranging trial of etoricoxib in patients with osteoarthritis. Rheumatology (Oxford). 2002;41:1052-61

5. Curtis SP, Mukhopadhyay S, Ramey D, Reicin AS. Etoricoxib cardiovascular safety summary. Circulation. 2003;108:378-9.

6. Guccione AA, Felson DT, Anderson JJ, et al. The effects of 
specific medical conditions on the functional limitations of elderinFraminghamStudy. AmJPublic Health. 1924;84:351-58.

7. American College of Rheumatology Subcommittee on Osteoarthritis Guideline. Recommendations for the medical management of osteoarthritis of the hip and knee. Arthritis Rheum. 2000;43:1905-15.

8. Bradley JD, Brand KD, Katz BP, et al. Comparison of an anti-inflammatory dose of ibuprofen, an analgesic dose of ibuprofen and acetaminophen in the treatment of patients with osteoarthritis of the knee. N Engl J Med. 1991;325:91.

9. McAlindon $\mathrm{T}$, Dleppe $\mathrm{P}$. The medical management of osteoarthritis of the knee: an inflammatory issue? Br J Rheumatol. 1990;29:471-3.

10. Wolfe MM, Lichtenstein DR, Singh G. Gastrointestinal toxicity nonsteroidal anti-inflammatory drugs (published correction appears in N Engl J Med 1991; 341:548) N Engl J M. 1999;340:1888-99.

11. Palmoski MJ, Brandt KD. Effects of some non-steroidal anti-inflammatory drug on proteglycan metabolism and organization in canine articular cartilage. Arthritis Rheum. 1980;23:1010-20.

12. FitzGerald GA Patrono C. The Coxibs, Selective inhibitors of cyclooxygenase-2. N Engl J Med. 2001;345:433-42.

13. Pasero G, Marcolongo R, Semi U, Pamhem JH, Fene F. A review: Study-Curr Med Res Opin. 1995;13(6):305-15.
14. Register JY, Paul I, Henrotin Y. Role of Aceclofenac in therapeutic arsenal against chronic osteoarthritis, pathogenesis. Rev Med Liege. 2001 July;56(7):484-8.

15. Kornassoff D, Frerick H, Bowdler J, Montull E. Aceclofenac is a well tolerated alternative to Naproxen in treatment of osteoarthritis. Clin Rheumatol. 1997 Jan;16(1):32-8.

16. Maneiro E, Lopez-Armada MJ, Fernanandez Sueiro JL, Lema B, Galdo F, Blanco FJ. Aceclofenac increases the synthesis of interteukin 1 receptor antagonist and decreases the production of nitric oxide in human articular chondrocytes. J Rheumatol. 2001 Dec;28(12):2692-9.

17. Scott DL and Paimer RH. Safety and efficacy of nabumetone in osteoarthritis: Emphasis on gastrointestinal safety ailment. Pharmacol Ther. 2000;14:443-52.

18. Gijon Banos J. Efficacy and safety of nabumetone in the treatment of knee osteoarthritis: a comparative clinical trial versus aceclofenac. Study Group of Nabumetone for Osteoarthritis of knee. Med Clin (Barc). 1997 Jun 21;109(4):1304 .

19. Morgan GJ Jr, Kaine J, Delapp R, Raimer R. Treatment of elderly patients with nabumetone or diclofenac: GI safety profile. J Clin Gastroenterol. 2001 Apr;32(4):310-4.

20. Fleischmann RM, Flint K, Constantine G, Kolecki B. A double- masked comparison of Napralen and nabumetone in osteoarthritis of the knee: Napralen Study Group. Clin Ther. 1997 Jul- Aug;19(4):642-55. 